\title{
Forming Clusters from Census Areas with Similar Tabular Statistics
}

\author{
Murray A. Jorgensen \\ Department of Statistics, University of Waikato \\ Hamilton, New Zealand \\ maj@waikato.ac.nz \\ June 17, 2011
}

\begin{abstract}
This paper presents a methodology for the clustering of a large number of tables of similar form. Data of this kind is often available from National Statistical Offices as tabulations of a set of variables for each of a large number of geographic units. Model-based clustering is employed, the underlying model being a finite mixture of multinomial distributions. We discuss the choice of the number of clusters and the interpretation of the clusters found. As a case study we consider a set of age-group by sex tables for small areas obtained in the New Zealand Census of Population and Dwellings for the year 2006.
\end{abstract}

\section{Introduction}

National statistical offices often now make available tabular data on census variables for fairly small geographical regions. The United Kingdom National Statistics Office has a web site www.neighbourhood.statistics.gov.uk from which tables at the post code level may be ob- 
tained. Similarly Statistics New Zealand provides regional tables through "Table Builder" at www.stats.govt.nz/tools_and_services/tools/TableBuilder.aspx.

The most common way in which such web sites are used is for the user to select a single area or a relatively small number of areas of greatest interest to him or her. When there is a more general interest in the variables across a country or state the user is faced with the problem of digesting information from perhaps thousands of contingency tables. If it is possible to group the small areas for which data is available into larger regions such that the cell proportions in the tables for areas within a region are similar this will be of great value for an exploratory analysis of the data. The problem with tables for large geographical regions is that they combine smaller regions for which the relationships between the variables may be quite different leading to a misleading picture in the aggregated table. (Essentially the basis of Simpson's Paradox.)

Most clustering methodologies rely on having information recorded at the individual level. Now while Statistics Offices are increasingly making available synthetic or confidentialized unit record data there will probably always be more data available in tabular form. In this article we illustrate the application of a clustering method appropriate for clustering small areas in such a way that areas with similar cell proportions in an associated contingency table will tend to be grouped together.

\section{Age by Sex tables}

As an example we will consider the tables of the variables Agegroup and Sex at the 2006 New Zealand Census of Population and Dwellings. This data may be obtained from the Statistics New Zealand Site mentioned above for different sizes of Geographic Unit. The geographic unit chosen was the "area unit", roughly equivalent to the US Census "block group" and averaging somewhat under 3000 population. It is convenient to think of an area unit as a small town or suburb, although they do not necessarily correspond to real social units.

As an example consider the area unit named Raglan, which corresponds roughly to the small town of the same name on the west coast of the North Island of New Zealand. The population 
Table 1: Raglan population by age and sex at the 2006 Census

\begin{tabular}{lrrrrrrrrr}
\hline \hline Agegroup & $0-4$ & $5-9$ & $10-14$ & $15-19$ & $20-24$ & $25-29$ & $30-34$ & $35-39$ & $40-44$ \\
\hline Male & 96 & 96 & 111 & 93 & 54 & 81 & 96 & 78 & 81 \\
Female & 81 & 90 & 84 & 87 & 54 & 81 & 117 & 105 & 105 \\
\hline \hline Agegroup & $45-49$ & $50-54$ & $55-59$ & $60-64$ & $65-69$ & $70-74$ & $75-79$ & $80-$ & Total \\
\hline Male & 99 & 84 & 69 & 66 & 48 & 36 & 42 & 24 & 1272 \\
Female & 96 & 93 & 81 & 63 & 60 & 48 & 48 & 42 & 1365 \\
\hline
\end{tabular}

counts obtained for this area unit as extracted from the web site are given in Table 1.

\subsection{Random Rounding}

A glance at Table 1 shows that all entries are divisible by 3 . This is clearly by artifice, not chance and is a consequence of the method of random rounding which has been applied to the counts before they are made available by Statistics New Zealand. In this method as used in these census tables counts that are not multiples of 3 are perturbed by rounding either to the closest multiple of 3 (with probability $\frac{2}{3}$ ) or to the second-closest multiple of 3 (with probability $\frac{1}{3}$ ). More confidentiality but less accuracy may be obtained by similar schemes where the rounding is to numbers divisible by 5 or by 10 . The same rounding scheme is applied to the margins of the table so that they need not agree with the appropriate totals of the rounded cell counts.

This feature is often felt to be undesirable and a related method known as controlled rounding ensures that the summation relations between the perturbed cell counts and margins are respected. Both rounding methods are discussed by Willenborg and de Waal (2000) and Federal Committee on Statistical Methodology (2005) where further references are given.

From the point of view of statistical modelling any system of controlled rounding must introduce probabilistic dependence into the data and the independent perturbations of random rounding would be preferred. It might also be argued that the tables produced by random rounding are less likely to be mistakenly treated by the general user as exact counts if the 
failure of marginal totals to be "correct" is noticed.

In this article we naively model the rounded counts as if they were actual counts, but the "missing information" approach of the EM algorithm could be extended to handle the information missing due to rounding. A 30-cell table randomly rounded to counts that are multiples of 3 could, if it contains no zeros, have arisen from any one of $5^{30}$ tables of actual counts. Combinatorial considerations like this make the application of EM to all rounded cells of such a large table unfeasible. However a few cells could be handled and in certain circumstances this could be an alternative to combining cells.

When summarizing census data the choice of the size of geographic unit to be used in tabulations thus relies on a trade-off:

- If too small a unit is used, confidentiality procedures applied by the National Statistics Office will introduce relatively large errors into the tables.

- If too large a unit is used, many subunits with quite different cell proportions will be merged, destroying information.

With the size of units selected in our case study no doubt both problems still remain but hopefully neither problem dominates.

\subsection{Preliminary Processing}

To reduce the number of parameters in the models to be fitted some of the older age classes were combined resulting in the new groups ' $65-74$ years' and ' 75 or older'. The later was calculated by subtraction from the margin and a few negative derived counts were replaced by zero. The resulting tables are now 15 by 2 in dimension. To reduce the effect of random rounding on the analyses area units with total count less than 600 (i.e. mean cell count less than 20) were omitted. There were 1455 area units included in the analysis containing over $97 \%$ of the New Zealand population. 


\section{Mixture Model Clustering}

We now seek to cluster area units into groups of similar demographic (age by sex) structure. The method that we use is based on finite mixture models. The description of these models may be given fairly succinctly as follows.

A finite mixture model is a probability distribution of the form

$$
p(y)=\sum_{j=1}^{q} \pi_{j} p_{j}(y)
$$

where for $j=1, \ldots, q$ the $\pi_{j}$ are non-negative proportions summing to 1 , and the $p_{j}$ are probability distribution functions. Usually the $p_{j}$ are assumed to come from the same parametric model family. The pdf $p(y)$ may be seen as a mixture of the pdf's $p_{j}(y)$ in proportions $\pi_{j}$. The connection with clustering is that if $y$ is a random observation from the population described by $p(y)$, the probability that $y$ comes from the subpopulation described by $p_{j}(y)$ is

$$
z_{j}=\frac{\pi_{j} p_{j}(y)}{\sum_{j=1}^{q} \pi_{j} p_{j}(y)} .
$$

If the subpopulations are well-separated and well-fitted by the $p_{j}$ then it is usually the case that one of the $z_{j}$ is close to 1 and the others are close to zero. In these situations the rounded $z_{j}$ act as indicator variables for the $q$ clusters.

It is necessary to choose a value for $q$. Clearly we might adopt a model with one cluster for each area unit and set $q=n$ but this achieves no data reduction at all and consequently no insight. The choice of $q$ is discussed in section 6.

If the $p_{j}$ are chosen from an exponential family it is straightforward to apply the EM algorithm (Dempster et al., 1977; McLachlan and Krishnan, 1997) to estimate both the $\pi_{j}$ and the parameters in the $p_{j}$ by maximum likelihood. The $z_{j}$ are estimated along with the parameters as part of the algorithm, and form the basis for the assignment of individual observations to clusters.

One form of unidentifiability is always present in mixture models: a permutation of the group labels leaves the likelihood unchanged so that any likelihood maximizing parameter vector shares this property with all its group-label permutations. This is not usually of concern in 
clustering applications and a convention may be adopted to order the groups according to the ordering of some function of the parameters. Some problems remain. For example in resampling methods such as the bootstrap the appropriate ordering of the groups may not be preserved under resampling. More local methods such as the infinitesimal jackknife would be more appropriate for inference about parametric functions not invariant under group-label permutations. Another type of unidentifiability arises when the number of mixture components $q$ is large in relation to the number of observations $n$. Portela (2008) discusses this and concludes that this will not occur if $q \leq(n+1) / 2$. This condition is far from being violated by the models that we consider in this paper.

Although the use of mixture models in many applications of statistics has grown rapidly in recent years they are relatively novel in Official Statistics. A recent example of their use in Official Statistics is the work of Di Zio et al. (2007) on missing data imputation.

\section{Mixtures Of Multinomial Distributions}

The discussion above applies generally to all types of mixture models but we will now consider models appropriate for clustering tabular data from a large number of geographic units.

We will be considering data where each observation is a table consisting of counts in each of a number of categories. In the case of the Age by Sex study we have, for each of the $n=1455$ area units, $p=30$ counts corresponding to the total number of 2006 residents of that area unit in each of the $30=15 \times 2$ demographic categories used in the study.

We now pose the question as to whether the great bulk of the area units fall into one of a finite number of patterns as far as their demographic structure is concerned. The observed category counts for a particular area unit might then be regarded as a sample from one of $q$ 30-category multinomial distributions.

Consider the following model for the probability $p_{i}\left(y_{i}\right)$ that the $i$ th area unit has counts of $y_{i}=\left(y_{i 1}, y_{i 2}, \ldots, y_{i p}\right)^{\prime}$ in the $p=30$ demographic categories

$$
p_{i}\left(y_{i}\right)=p_{i}\left(y_{i 1}, y_{i 2}, \ldots, y_{i p}\right)
$$




$$
\begin{aligned}
& =\sum_{j=1}^{q} \pi_{j} \frac{m_{i} !}{y_{i 1} ! y_{i 2} ! \cdots y_{i p} !} \lambda_{j 1}^{y_{i 1}} \lambda_{j 2}^{y_{i 2}} \cdots \lambda_{j p}^{y_{i p}} \\
& =\sum_{j=1}^{q} \pi_{j} p_{i j}\left(y_{i}\right)
\end{aligned}
$$

where $m_{i}$ stands for $y_{i 1}+y_{i 2}+\cdots+y_{i p}$ and the mixing proportions $\pi_{j}$ sum to 1 .

This is essentially a finite mixture of multinomial distributions $\operatorname{Multi}\left(m_{i}, \lambda_{j 1}, \lambda_{j 2}, \ldots, \lambda_{j p}\right)$, except that the numbers $m_{i}$ vary depending on the area unit $i$. Mixture modelling seeks to identify subclasses in the data of distinctly different structure. In the present context we may hope that the $q$ subclasses in the mixture model will correspond to different kinds of community.

\section{The EM Algorithm}

The $E M$ algorithm, as applied to parameter estimation of finite mixture models, searches for maxima of the usual likelihood function, but it does so not directly but using another likelihood function. This other likelihood function is based on unobserved indicator variables for the $q$ subpopulations as well as the observed categorized resident counts for each area unit.

It is convenient to set up an $n \times q$ matrix of indicator variables $Z=\left(z_{i j}\right)=\left(z_{i}^{\prime}\right)$ where $z_{i j}=1$ if case $i$ belongs to subclass $j$ and otherwise is zero. These unobserved indicator variables play a role in the $E M$ fitting algorithm and their estimates are a basis for assigning observations to clusters.

The observed data likelihood is the likelihood based on $y_{i k}$ and is given by

$$
\begin{aligned}
L & =\prod_{i=1}^{n}\left[\sum_{j=1}^{q}\left(\pi_{j} \frac{m_{i} !}{y_{i 1} ! y_{i 2} ! \cdots y_{i p} !} \prod_{k=1}^{p} \lambda_{j k}^{y_{i k}}\right)\right]=\prod_{i=1}^{n} p_{i}\left(y_{i}\right) \\
\text { whence } l & =\log L=\text { constant }+\sum_{i=1}^{n} \log p_{i}\left(y_{i}\right) .
\end{aligned}
$$

The likelihood based on $y_{i k}$ and $z_{i j}$ is given by

$$
L_{C}=\prod_{i=1}^{n} \frac{m_{i} !}{y_{i 1} ! y_{i 2} ! \cdots y_{i p} !} \prod_{j=1}^{q} \pi_{j}^{z_{i j}}\left(\prod_{k=1}^{p} \lambda_{j k}^{y_{i k}}\right)^{z_{i j}} .
$$


Taking logs and omitting the constant:

$$
l_{C}=\sum_{i=1}^{n} \sum_{j=1}^{q}\left[z_{i j} \log \pi_{j}+\sum_{k=1}^{p} y_{i k} z_{i j} \log \lambda_{j k}\right] .
$$

This is the complete-data log-likelihood in EM terminology. The EM algorithm itself for this estimation problem is defined by two steps:

$\boldsymbol{E}$-step Taking $\pi$ and $\lambda$ as given; estimate $Z$.

M-step Taking $Z$ as given; estimate $\pi$ and $\lambda$.

which in our case leads to

$$
\begin{array}{ll}
\text { E-step: } & \hat{z}_{i j}=\frac{\pi_{j} p_{i j}\left(y_{i}\right)}{p_{i}\left(y_{i}\right)} . \\
\text { M-step: } & \hat{\pi}_{j}=\frac{\sum_{i} z_{i j}}{n} ; \quad \hat{\lambda}_{j k}=\frac{\sum_{i} y_{i k} z_{i j}}{\sum_{i} m_{i} z_{i j}} .
\end{array}
$$

The E-step essentially estimates the unknown quantities in the complete-data log-likelihood, taking the current parameter values to be the true ones. The $\log$-likelihood $l(\pi, \lambda)=\log \sum_{i=1}^{n} p_{i}\left(y_{i}\right)$ comes almost free as a byproduct of the E-step.

The M-step maximizes the so-estimated complete-data log-likelihood function to find an improved set of parameter estimates. The theory of the EM algorithm (Dempster et al., 1977; McLachlan and Krishnan, 1997) shows that a cycle of E-step plus M-step cannot decrease the likelihood.

\subsection{EM outputs}

The $E M$ algorithm delivers estimates of the $p=30$ cell probabilities for each of the $q$ subpopulations, as well as the $q$ mixing proportions. By construction each set of $p$ cell probabilities sums to 1 as do the $q$ mixing proportions.

Also, and of direct relevance to the goal of identifying clusters, the EM algorithm produces the $\hat{z}_{i j}$, estimates of the probability that area unit $i$ belongs to cluster $j$. 
The $i$ th term $l_{i}$ of the observed data $\log$-likelihood $l$ (in other words the logged value of the mixture density for the $i$ th area unit according to the current parameter values), is calculated in the course of calculating the $\hat{z}_{i j},(i=1, \ldots, n)$ so that $l$ is available for each fit.

The natural way to use the $\hat{z}_{i j}$ is to associate each area unit to its most probable cluster, although it might be reasonable to leave some area units unassigned unless their largest $\hat{z}_{i j}$ exceeded some threshold.

An alternative is to use a probabilistic assignment in which area unit $i$ is assigned to cluster $j$ with probability $\hat{z}_{i j}$. Although probabilistic assignment may not be optimal for a particular area unit it shows the shape and extent of a cluster better.

If $Z$ is the $n \times q$ matrix with $i, j$ element $\hat{z}_{i j}$ then the matrix $Z^{\prime} Z$ has as its $j, k$ element the expected number of area units assigned to cluster $j$ on one probabilistic assignment and assigned to cluster $k$ on another independent assignment $(j, k=1,2, \ldots, q)$. It is desirable that the off-diagonal elements of this matrix are small. The larger off-diagonal elements indicate which pairs of clusters are 'close' or 'overlapping'. In this paper we will call this the overlap matrix.

\section{Choosing the Number of Clusters}

In the present case study should not be thought that an integer $q$ can be identified which is the 'true' number of clusters that the census area units should be grouped into. Even if there were, in fact, a finite number of regions such that tables for variables describing small connected areas contained in a region could be treated as random samples from a regional multinomial distribution this would not imply that the area units are all such samples. This is because area unit boundaries are not chosen with such regions in mind and inevitably many would straddle two or more regions.

In the present case where the variables under study are sex and age group a more specific argument can be given. Consider the structure of the Raglan population in Table 1. Note that the number of residents in their twenties is low compared to those immediately younger 
or older. This bi-modal pattern is quite common in area units and in many cases reflects a preponderance of nuclear family households with children living with their parents. However the location of the 'parent' and 'child' modes differs between area units according to whether the unit is dominated by younger or older families at the time of the census. A finite mixture model will assign such nuclear-family dominated area units into a finite number of clusters. Clearly, however, the underlying reality is that of a continuous mixture of family ages to which the finite mixture merely provides an approximation.

So what we are trying to do when fitting a mixture model is to identify a small number of typical patterns of cell probabilities that approximates the very much larger set of cell relative frequencies in the data.

The simplest way to evaluate the quality of the approximation is via the observed data loglikelihood $l$. Various information criteria are also used in the literature and a good survey in the mixture model context is given by McLachlan and Peel (2000, sections 6.8,6.9). These include the Classification Likelihood Criterion, $l-e$, the Bayesian Information Criterion, $l-d \log (n) / 2$, and the Integrated Classification Likelihood Criterion $l-d \log (n) / 2-e$. We refer to these criteria respectively as CLC, BIC and ICL. The CLC and the ICL were introduced by Biernacki et al. (1999). Here $q$ is the number of mixture components in the model, $n=1455$ is the number of area units, $d=q-1+q(p-1)=p q-1$ is the number of independent model parameters to be estimated, and $e=\sum_{i} \sum_{j} \hat{z}_{i j} \log \left(\hat{z}_{i j}\right)$ is known as the entropy, and is small when each area unit is assigned to a single cluster with high probability.

Various numbers of mixture components $q$ were investigated in the following way. For each value of $q, 100$ random partitions of the area units into $q$ clusters were generated and the $E M$ algorithm run with these as the starting clusters. The solution with the greatest log-likelihood of the 100 fits was chosen. In Table 2 we give likelihood and information statistics for a number of model fits having a range of component numbers.

In Figure 1 the log-likelihood $l$ and the ICL are plotted against the number of mixture components in the model. The BIC and the CLC lie in between these two curves. 
Table 2: Likelihood and information statistics for several fits

\begin{tabular}{rrrrr}
\hline \hline$q$ & $l$ & $d$ & $e$ & $\mathrm{ICL}$ \\
\hline 3 & -136505.70 & 89 & 15.12 & -136844.9 \\
6 & -94221.55 & 179 & 23.75 & -94897.11 \\
9 & -81314.63 & 269 & 38.9 & -82333.06 \\
12 & -71283.36 & 359 & 47.29 & -72637.91 \\
15 & -64616.77 & 449 & 56.74 & -66308.49 \\
18 & -61488.93 & 539 & 62.52 & -63514.15 \\
21 & -58187.13 & 629 & 62.84 & -60540.39 \\
24 & -55474.69 & 719 & 65.88 & -58158.72 \\
27 & -54016.51 & 809 & 88.66 & -57051.05 \\
\hline
\end{tabular}

The ICL is still climbing at $q=27$ which is rather remarkable as the 27 -component model has 809 parameters!

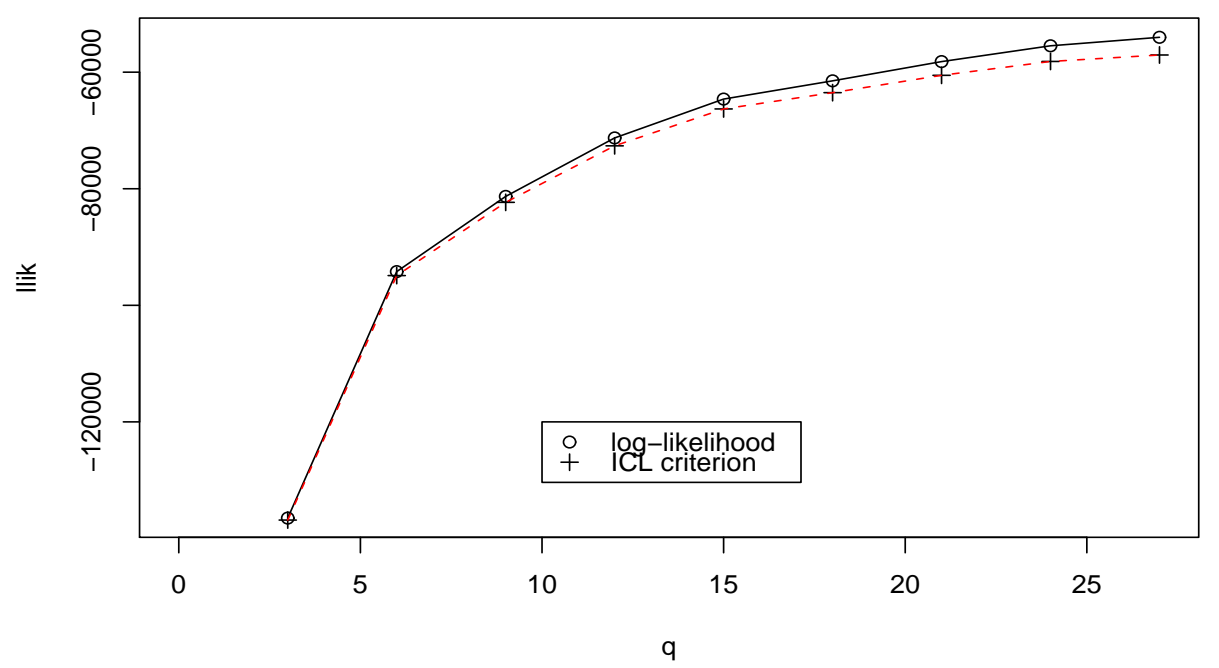

Figure 1: Log likelihood versus number of components 
The reason for the usual criteria favouring a very large number of mixture components seems to be that the multinomial distribution is underdispersed for use in this way, at least for the data being considered. It may be worth exploring more dispersed component distributions than the multinomial such as the Dirichlet-multinomial as in principle it would require fewer of such components to approximate the distribution of the data. We will not pursue this approach here.

From Figure 1 it appears that the 12-component solution is a good compromise between fit and complexity and we choose this solution to describe more fully here for the sake of brevity.

\section{Diagnostics}

As mentioned in section 5.1, the $E M$ algorithm provides the value of the fitted density function at each area unit. Area units fitted poorly by the model will have relatively low values of this density. The density values turn out to be very skew indeed and we recommend transforming by $\log (-\log (\cdot))$ to produce quantities that could be regarded as residuals for the area units.

In Figure 2 we display boxplots of $\log (-\log ($ density $))$ values for each cluster. Notice that Clusters A, B, and $\mathrm{H}$ are relatively poorly fit. We will discuss this further below.

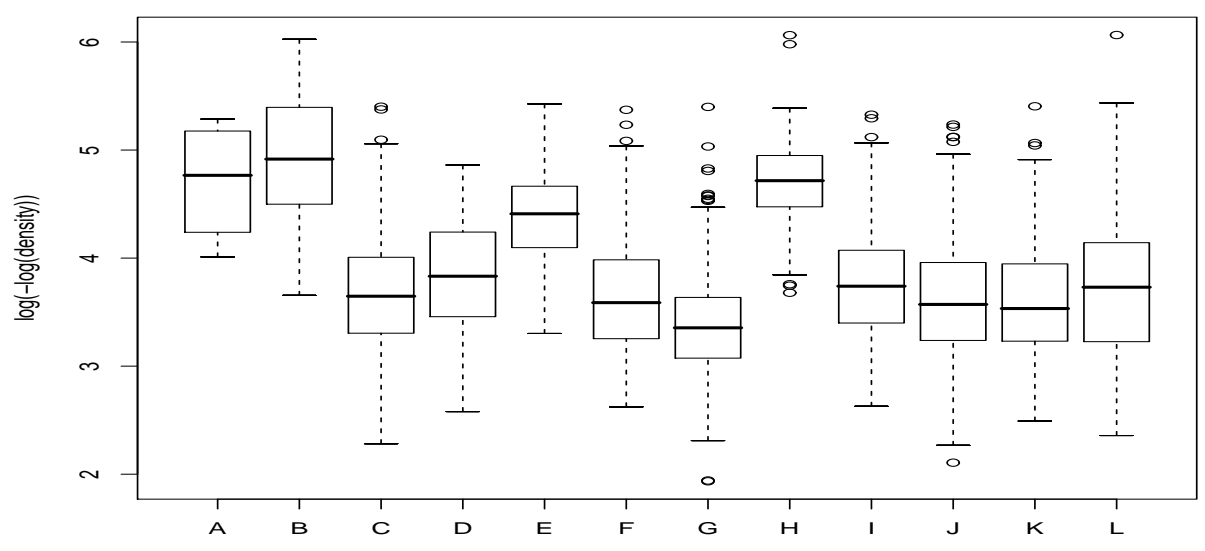

Figure 2: Boxplots of $\log (-\log ($ density $))$ by Cluster 


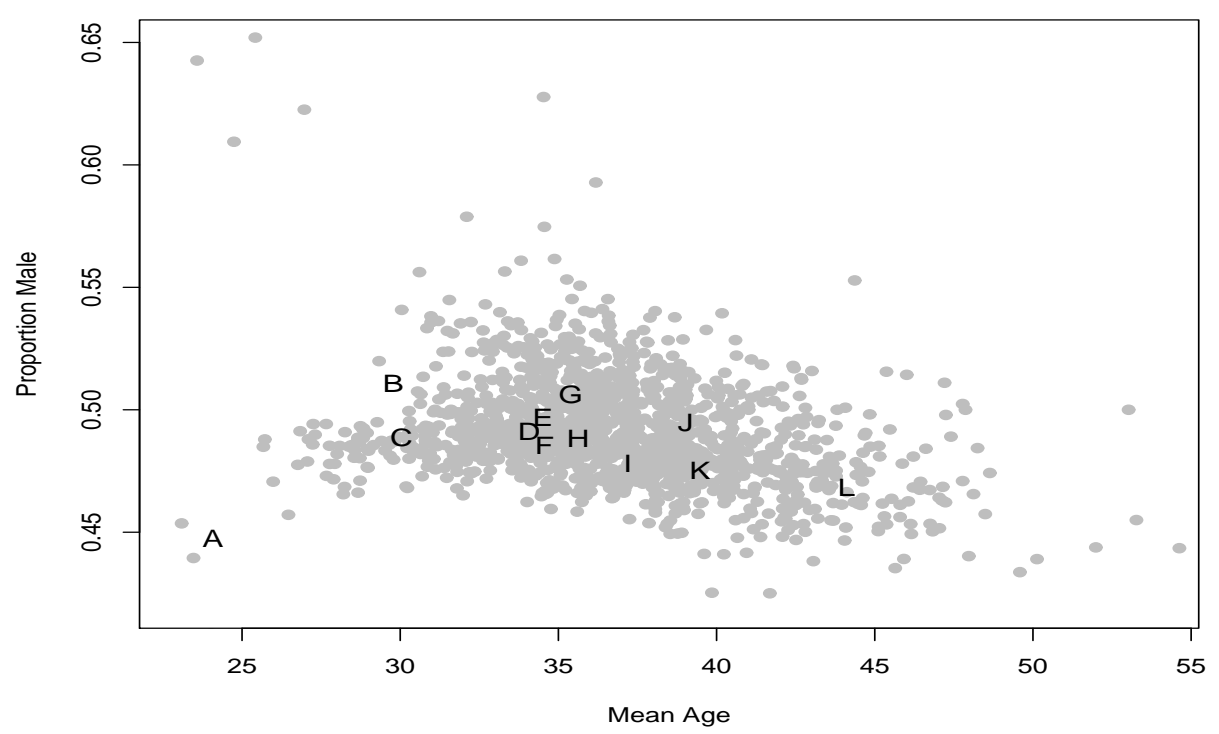

Figure 3: Proportion Male and Mean Age for Area Units and Clusters

\section{Description of the 12-Cluster Fit}

Figure 3 contains a plot of the proportion of males in an area unit against the mean age of the area unit. For the purpose of calculating mean age the mid-points of the age groups were used, except for the "75 and over" group, for which a mean age of 80 was used. Three outlying area units having values $(54.6,0.443),(24.0,0.409)$ and $(41.5,0.739)$ were omitted from the plot.

The clusters are labeled ' $A$ ' through to ' $L$ ' in order of increasing mean age. Using these letters as plotting symbols the values for the proportion of males and mean age as derived from the estimated cluster parameters have been added to the plot.

To complement the visualization of Figure 3 we also present (Table 3) the overlap matrix described in section 5.1, rounded to integers for convenience. The most noticeable overlap is between clusters $\mathrm{G}$ and $\mathrm{J}$, which appear reasonably separated on the plot. In contrast clusters D, $\mathrm{E}$, and $\mathrm{F}$ which plot quite closely show no overlap to the precision printed. (The largest overlap is 0.4 between $\mathrm{D}$ and F.) These differences between the plot and the table remind us that the plot is only a particular 2-dimensional projection of a much higher dimensioned structure. 
Table 3: Overlap matrix for 12-cluster fit

\begin{tabular}{|c|c|c|c|c|c|c|c|c|c|c|c|c|}
\hline & A & $\mathrm{B}$ & $\mathrm{C}$ & $\mathrm{D}$ & $\mathrm{E}$ & $\mathrm{F}$ & $\mathrm{G}$ & $\mathrm{H}$ & $\mathrm{I}$ & $\mathrm{J}$ & $\mathrm{K}$ & $\mathrm{L}$ \\
\hline A & 4 & . & & . & . & . & . & . & & . & . & . \\
\hline B & . & 17 & & . & . & . & . & . & & . & . & . \\
\hline C & & & 121 & . & . & 1 & . & & . & . & . & . \\
\hline D & . & & $\cdot$ & 91 & . & . & . & & 1 & & . & . \\
\hline E & . & . & . & . & 38 & . & . & . & . & . & . & . \\
\hline F & . & . & 1 & & & 178 & 2 & . & . & 1 & . & . \\
\hline G & . & . & . & & & 2 & 284 & . & . & 4 & . & \\
\hline $\mathrm{H}$ & . & & . & . & . & . & . & 23 & . & . & . & . \\
\hline I & . & & . & 1 & . & . & . & . & 128 & . & 1 & . \\
\hline $\mathrm{J}$ & . & . & . & . & & 1 & 4 & . & . & 229 & 1 & 1 \\
\hline $\mathrm{K}$ & . & . & . & & & & . & . & 1 & 1 & 183 & 1 \\
\hline L & . & . & . & . & . & . & . & . & . & 1 & 1 & 130 \\
\hline
\end{tabular}

Next, Table 4 gives information on the proportion of males and the mean ages by cluster and the proportions of area units and total population that belong to each cluster. These are not of great interest in themselves as it is the distribution of males and females across the age-group categories that is of most interest and we now turn to this.

Table 4: Summary statistics for 12-cluster fit

\begin{tabular}{lcccrcr}
\hline \hline Cluster & $\begin{array}{c}\text { Prop. } \\
\text { Male }\end{array}$ & $\begin{array}{c}\text { Mean } \\
\text { Age }\end{array}$ & $\begin{array}{c}\text { Prop. } \\
\text { Units }\end{array}$ & $\begin{array}{c}\text { No. } \\
\text { Units }\end{array}$ & $\begin{array}{c}\text { Prop. } \\
\text { of Pop. }\end{array}$ & $\begin{array}{c}\text { Pop. } \\
(000 \mathrm{~s})\end{array}$ \\
\hline A & 0.448 & 24.082 & 0.003 & 4.000 & 0.003 & 12.144 \\
B & 0.511 & 29.778 & 0.012 & 17.000 & 0.014 & 53.757 \\
C & 0.489 & 30.038 & 0.084 & 122.878 & 0.099 & 388.859 \\
D & 0.491 & 34.079 & 0.064 & 92.961 & 0.078 & 307.660 \\
E & 0.497 & 34.518 & 0.026 & 37.985 & 0.030 & 117.185 \\
F & 0.486 & 34.563 & 0.125 & 182.091 & 0.145 & 567.911 \\
G & 0.506 & 35.389 & 0.199 & 289.852 & 0.149 & 583.368 \\
H & 0.488 & 35.628 & 0.016 & 23.001 & 0.017 & 66.189 \\
I & 0.478 & 37.196 & 0.089 & 129.572 & 0.111 & 433.665 \\
J & 0.495 & 39.024 & 0.162 & 236.339 & 0.126 & 494.300 \\
K & 0.475 & 39.478 & 0.128 & 186.858 & 0.140 & 547.516 \\
L & 0.468 & 44.105 & 0.091 & 132.465 & 0.089 & 350.001 \\
\hline
\end{tabular}

Although the standard caveats about overhasty reification of artificially constructed groups or variables common to all multivariate analysis hold for this analysis it is useful to tentatively 
identify some of the clusters found with verbal descriptions.

We base our discussion primarily on the estimated cell proportions for each cluster but also on the names of the area units as assigned to the cluster of highest estimated probability. Unfortunately these area unit names for each cluster cannot be listed for reasons of space, but the author is happy to supply a file with this information.

In the plots of the cell proportions the points for males are connected by solid lines; those for females by dashed lines. Each age group except the highest two has a 5 year range. The second-highest age group represents 10 years and these proportions have been divided by 2 for plotting; the highest age group is open-ended and has been divided by 3 for plotting. The ' 3 ' was chosen after trial-and-error experimentation.

Some of the clusters seem to group together naturally and we take advantage of this to give a more compact description.

\subsection{Clusters A, B, and $\mathrm{H}$}

The cell proportions for these clusters are plotted in Figure 4. These three clusters share the property of having an age distribution strongly concentrated near 20. This is unique to these three and many of the other clusters have an antimode near 20. The area unit names for these clusters explain their odd composition: all are either universities, other tertiary institutions, military camps or adjacent suburbs.

The main distinction between the three clusters lies in how many non-student-age residents also reside in the area unit. Most of the residents of Cluster A live in or adjacent to the University of Otago in Dunedin. These areas are famous in New Zealand for "the Dunedin Sound" and "scarfie" student youth culture. It seems likely that the Cluster's uniquely concentrated demographic structure is related to this.

It is noteworthy that these three small clusters, though clearly separated from the rest of the clusters and from each other, show the poorest fit to the model. There is a case for removing them from the data and fitting the model to the remaining area units. We would certainly do this if we were considering fitting more mixture components for otherwise improvements in 


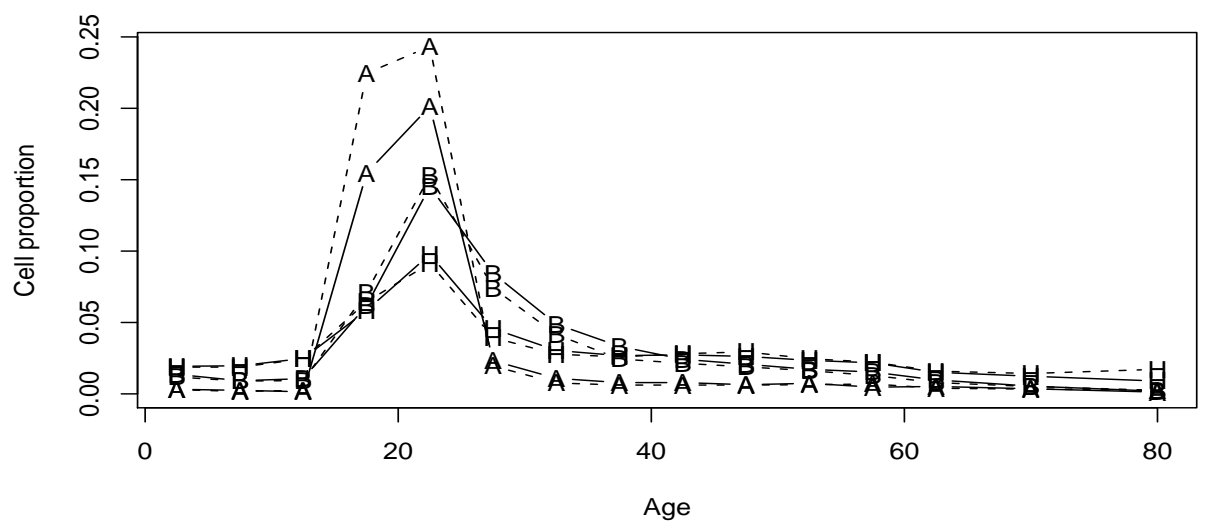

Figure 4: Cell proportions for Clusters A, B, and $\mathrm{H}$

the likelihood would be dominated by how well the fit to these area units improved. From the clustering viewpoint these clusters are well-understood and little would be gained by further subdivision of them, which a purely likelihood-driven approach would lead to.

\subsection{Clusters $\mathbf{C}$ and $\mathbf{F}$}

The cell proportions for these clusters are plotted in Figure 5.

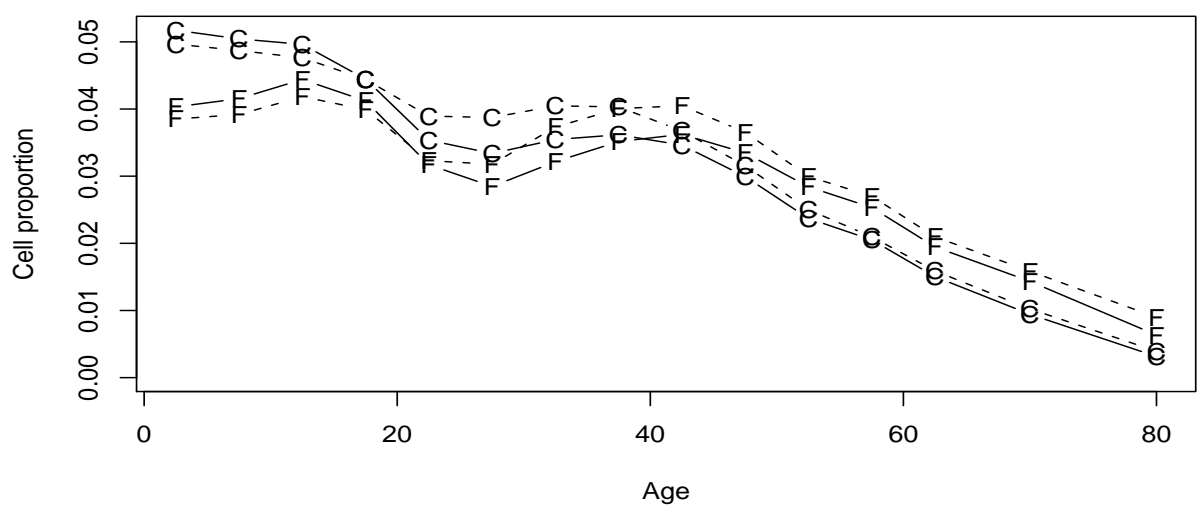

Figure 5: Cell proportions for Clusters $\mathrm{C}$ and $\mathrm{F}$ 
Leaving aside the rather artificial communities just discussed Cluster $\mathrm{C}$ is the cluster which contains New Zealand communities with the greatest number of children per adult. Included in this cluster are urban areas such as Otara and Cannons Creek with large Maori and other Polynesian populations. The only South Island area unit in this cluster is Rolleston, a dormitory suburb of Christchurch that has shown recent rapid growth.

Cluster F has a generally similar age profile to Cluster C but somewhat older. One might expect communities in Cluster $\mathrm{C}$ to naturally evolve into Cluster $\mathrm{F}$ communities over time.

\subsection{Clusters E and D}

The cell proportions for these clusters are plotted in Figure 6.

Cluster E has an age profile that reminds one of the "student clusters" but less concentrated and centered closer to 30 than 20 . The area units associated with this cluster are essentially the centers of the larger New Zealand cities. There are relatively few children and older people.

Taking these points together a colleague has suggested that E be named the "Yuppie" cluster.

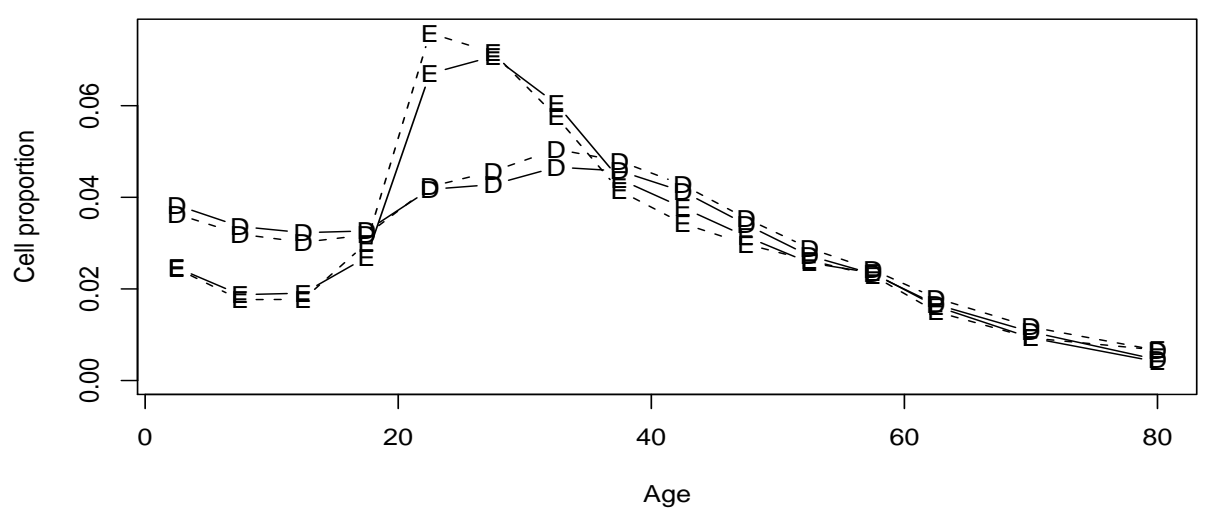

Figure 6: Cell proportions for Clusters E and D

Cluster D reflects a later stage in the life cycle than Cluster E, although its mean age has been lowered by the presence of more young children. Most area units making up this cluster are recognisably urban, but not quite as central as those in Cluster E. Typically they are inner 
suburbs. One might imagine families of Cluster E migrating to Cluster D as children begin to arrive.

\subsection{Clusters G and J}

The cell proportions for these clusters are plotted in Figure 7.

The distinguishing feature of these clusters is a strongly bimodal age profile with an antimode in the twenties. Also notable are the relatively low proportions of older residents. The area units in these clusters tend to be either rural areas or "lifestyle" outer suburbs of cities. These are not the sort of lifestyles that tend to interest young adults. The elderly would find the remoteness from medical assistance a problem.

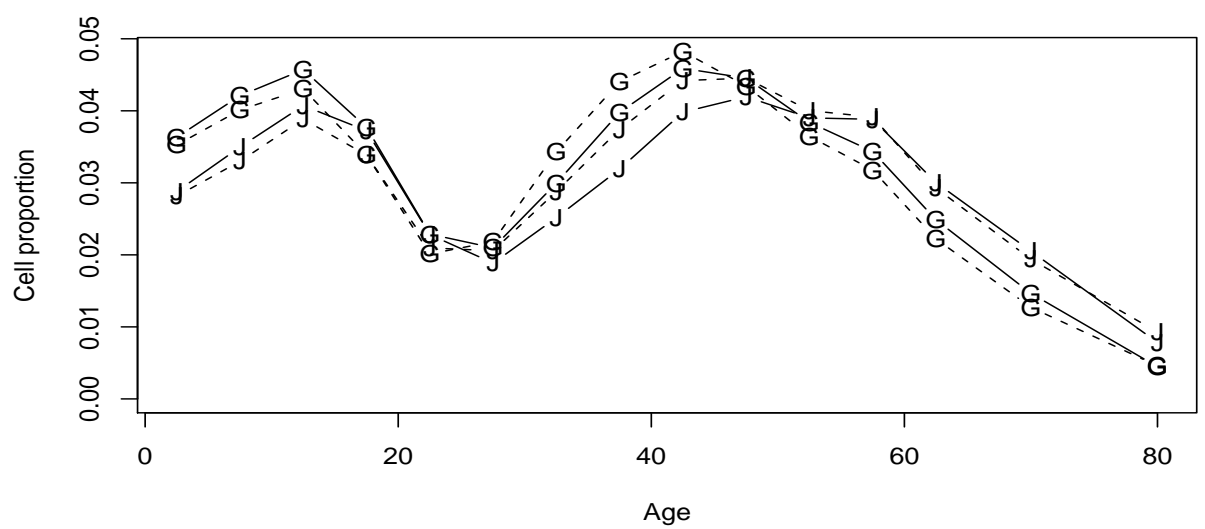

Figure 7: Cell proportions for Clusters $\mathrm{G}$ and $\mathrm{J}$

Cluster $\mathbf{J}$ has a very similar age profile to Cluster G but somewhat older. As noted in section 6 , Clusters $\mathrm{G}$ and $\mathrm{J}$ should probably be understood as representing a continuum of profiles of differing mean ages. 


\subsection{Clusters $\mathrm{K}$ and $\mathrm{L}$}

The cell proportions for these clusters are plotted in Figure 8.

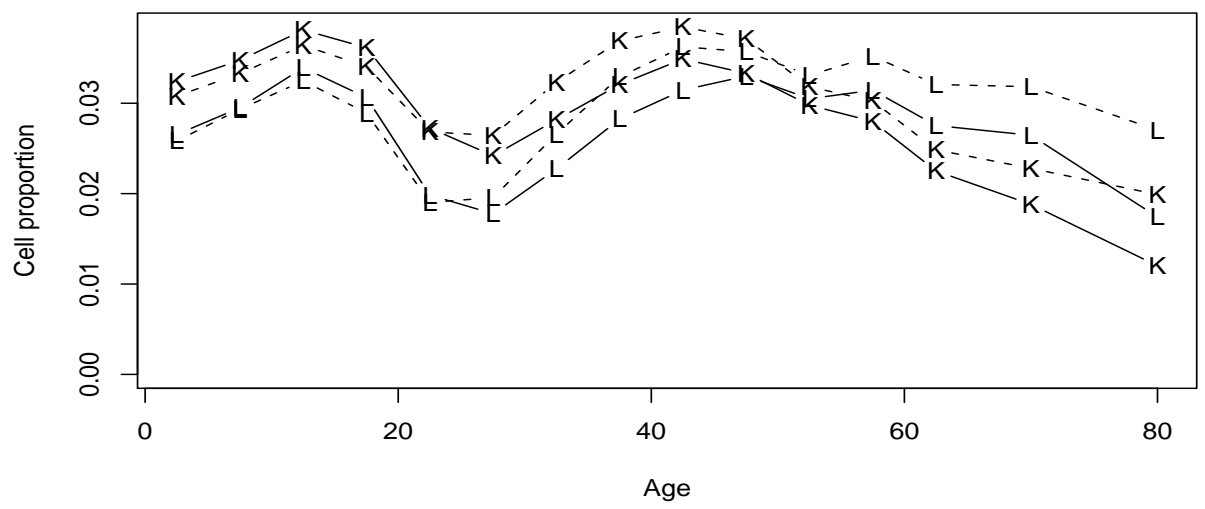

Figure 8: Cell proportions for Clusters $\mathrm{K}$ and $\mathrm{L}$

These clusters have the highest mean ages and the greatest proportions of the top two age groups among all the clusters. Ignoring the older age groups these clusters have the bimodal profile associated with nuclear families with a child mode at 12 and a parent mode around 42 . In some cases known to me the presence of a large rest home has modified the age profile of an area and moved it into one of these clusters.

Many traditional retirement areas can be found among the areas of these clusters but the areas tend not to be as isolated as those in Clusters G and J. Cluster L has a greater proportion of residents in the oldest two age groups than Cluster $\mathrm{K}$ but otherwise has a similar profile. This suggests that the elderly tend to migrate into Cluster L.

\subsection{Cluster I}

The cell proportions for this cluster are plotted in Figure 9.

The area units in this cluster are mostly suburbs in the larger cities. An unusual feature is that there is neither a large peak nor a big trough in the 20-24 age group so in this cluster 


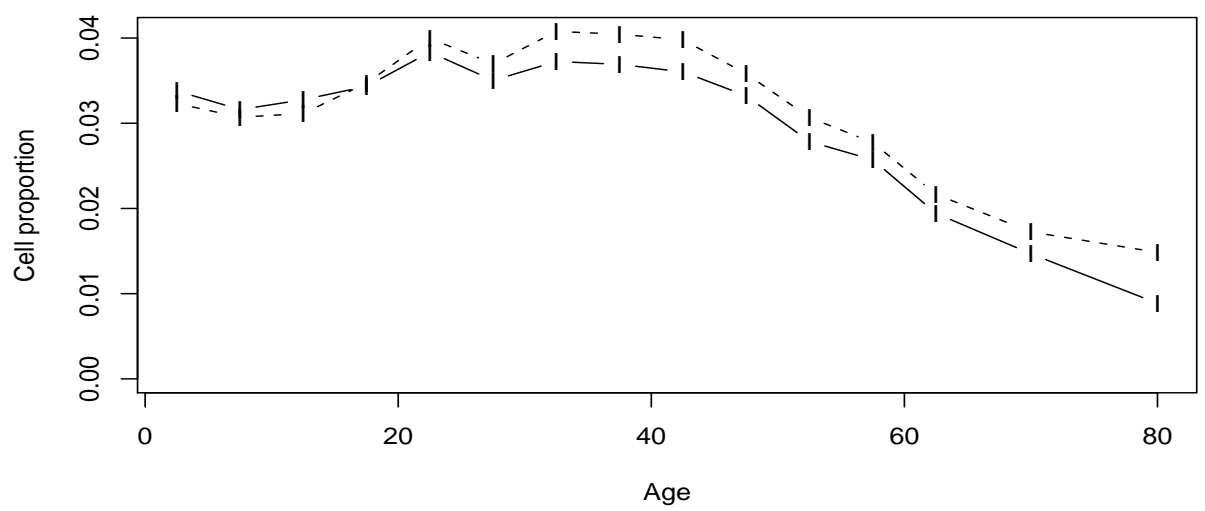

Figure 9: Cell proportions for Cluster I

a higher proportion than usual of young adults are living alongside children and older adults. For at least a proportion of these area units public transport is relatively good by New Zealand standards so that young adults can travel for education and employment while continuing to reside within or close to nuclear family households.

\section{Sex ratios}

The purpose of this article is to introduce a methodology for analysing census tables for small areas, and the actual tables studied (sex by age-group) are incidental to this purpose. However these two variables were chosen in the hope that the methodology could shed some light on one current phenomenon which is puzzling both providers and users of Official Statistics in New Zealand: a distinct majority of females over males in the 30-40 age range in the published census figures.

There is uncertainty and debate about whether this phenomenon is a real phenomenon or reflective of a gender-biased undercount (Bycroft, 2006). In contrast the male majority among the newborn and the female majority among the elderly are well-understood.

Consider, now, which of the groups of clusters show this female majority, focussing on the 
age groups centered at 32, 37, and 42. Firstly the "Student" clusters (A, B, and H) do not show the phenomenon: there are modest male majorities for these clusters. (Although in the age groups centered at 17 and 22 we see female majorities.) The "child-oriented clusters" (C and D) do show the female majority. The clusters E (urban) and D (inner-suburbs) go different ways with $\mathrm{E}$ showing a male, $\mathrm{D}$ a female, majority in the age groups of interest.The strongly bimodal clusters ( $\mathrm{G}$ and $\mathrm{J}$ ) show the female majority as do the older clusters (K and L) and Cluster I.

Is there a pattern in this? The only one that I am able to see is that it appears that the female majority is related to the care-giver role of many women in the 30-44 age range. To follow up on this we plot in Figure 10 the difference between the female and male proportions in the $30-44$ age group ("Female Majority") against the proportion in the age groups $0-9,75+$ which might be expected to contain the greater part of the population in need of care ("Proportion 0-9 \& 75+"). Three points have been omitted from the plot: $(0.21,-0.10),(0.19,-0.23)$, and $(0.20,-0.08)$.

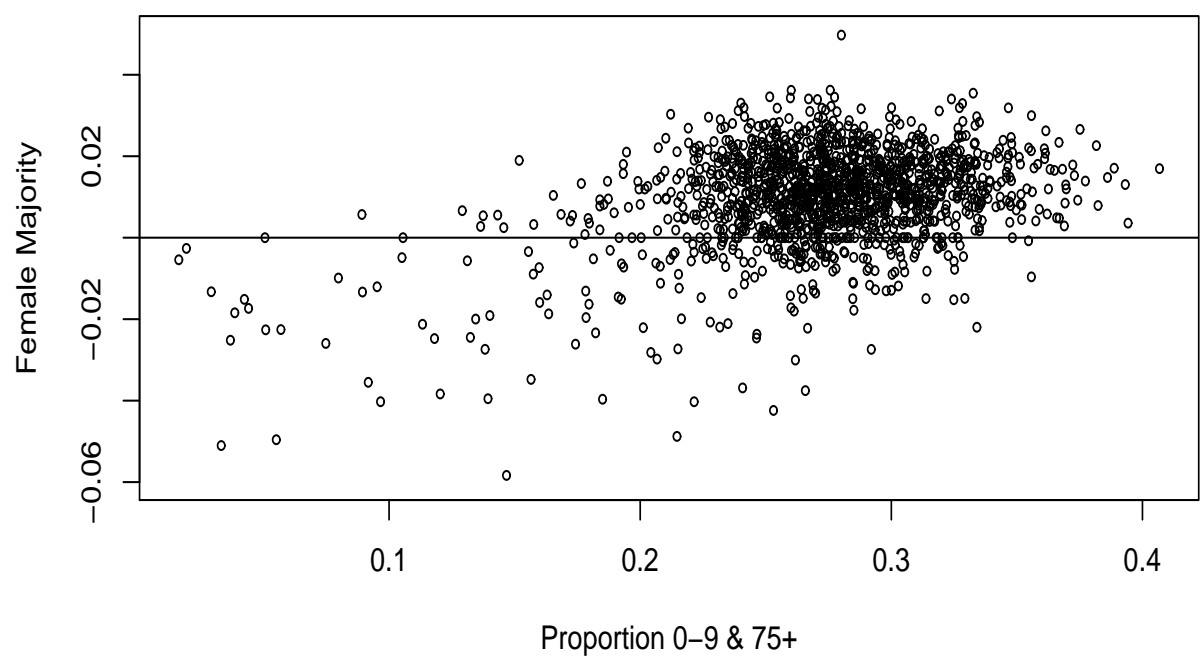

Figure 10: Female majority versus proportion of old and young

One explanation for the apparent positive association in this plot might be that (in this age 
range, in comparison to men) women are more closely bound to the extended family which tends to make them reside in localities with broad age distributions. (I do not suggest that that the closeness or otherwise of the extended family necessarily corresponds to the personal wishes of the individual man or woman.)

This does not in itself settle the question of whether the proportion of 30-44 year old males is truly low or so as a result of census undercount. For example it could be that some males avoid a caregiving role and also resist being enumerated for fear of official demands on their time or resources, not realising or trusting that New Zealand census returns are confidential.

It does suggest a way of resolving the question. Beginning with randomly chosen children and the elderly one could attempt to find the location of their close family members (parents or children). Problems in locating the male family members would suggest an undercount explanation; alternatively a greater proportion of male family members either deceased or overseas would suggest a true female majority.

\section{Conclusion}

Availability of census data at the level of small areas is welcome, but the resulting data can be difficult to study in an exploratory way. Grouping the areas into a moderate number of clusters with differing patterns of cell proportions provides a way to understand the "message" of the data, especially where the names and locations of the small regions are known to the analyst. A similar methodology was used by Jorgensen (2004) to cluster packet size distributions in packet flows over the internet between computers. That analysis was not as easy to interpret in the absence of background information about the various different packet flows.

Large Official Statistics data sets can be difficult for the user to assimilate because of the lack of a visual dimension. The methodology presented in this paper provides an approach to unlocking this key to understanding for large data sets. 


\section{References}

Biernacki, C., Celeux, G., and Govaert, G. (1999). An improvement of the NEC criterion for assessing the number of clusters in a mixture model. Pattern Recognition Letters, 20:267272.

Bycroft, C. (2006). Challenges in estimating populations. New Zealand Population Review, 32(2):21-47.

Dempster, A. P., Laird, N. M., and Rubin, D. B. (1977). Maximum likelihood from incomplete data via the EM algorithm (with discussion). J. Roy. Statist. Soc. B, 39:1-38.

Di Zio, M., Guarnera, U., and Luzi, O. (2007). Imputation through finite gaussian mixture models. Comput. Statist. Data Anal., 51:5305-5316.

Federal Committee on Statistical Methodology, . (2005). Report on statistical disclosure limitation methodology. Statistical Policy Working Paper 22 (Second Version), U.S. Office of Management and Budget, Washington, D.C.

Jorgensen, M. A. (2004). Using multinomial mixture models to cluster internet traffic. Austral. and New Zealand J. Statist., 46:205-218.

McLachlan, G. J. and Krishnan, T. (1997). The EM algorithm and extensions. Wiley, New York.

McLachlan, G. J. and Peel, D. (2000). Finite Mixture Models. Wiley, New York.

Portela, J. (2008). Clustering discrete data through the multinomial mixture model. Comm. Statist. Theory Methods, 37:3250-3263.

Willenborg, L. C. R. J. and de Waal, T. (2000). Elements of Statistical Disclosure Control, volume 155 of Lecture Notes in Statistics. Springer, New York. 